\title{
Obstructed tilting disc mitral valve prosthesis associated with placenta praevia
}

\author{
H. G. G. KINGSTON, B. T. LE ROUX, T. G. ARMSTRONG, AND F. MARGOLIS \\ From the Departments of Anaesthetics, Thoracic Surgery, Cardiology, and Obstetrics and \\ Gynaecology, University of Natal, Durban, Natal
}

Kingston, H. G. G., le Roux, B. T., Armstrong, T. G., and Margolis, F. (1977). Thorax, 32, 210-211. Obstructing tilting disc mitral valve prosthesis associated with placenta praevia. The occurrence of a bleeding placenta praevia in a young woman, with a viable fetus and an acutely obstructed Lillehei-Kaster valve, provided serious difficulties in priorities and decision making. This has prompted an analysis of the problems which others may encounter.

Successful pregnancy in women who harbour prosthetic heart valves is well documented (Bennett and Oakley, 1968; Barnard et al., 1969; Laros et al., 1970; Buxbaum et al., 1971; Reid and Barclay, 1971). Closed cardiac surgery in pregnancy was reported as long ago as 1952 (Brock, 1952; Logan and Turner, 1952). Later reports established that it was possible, in pregnancy, to undertake valve replacement with the adjunct of cardiopulmonary bypass with fetal survival. In a series of 20 cases, Zitnik et al. (1969) described a fetal mortality of $33 \%$, while the maternal mortality was $5 \%$, not higher than in non-pregnant patients. It is probable that the relatively non-pulsatile nature of blood flow during bypass, often with a low mean pressure, changing blood gas tensions, and disturbances of serum biochemistry, produces changes in uteroplacental perfusion and thus ultimately fetal oxygen supply, and thereby contributes to the high fetal mortality.

By a relatively simple technique of monitoring fetal heart rate with an ultrasonic transducer, Koh et al. (1975) were able to show that fetal distress, reflected by bradycardia, could in at least one instance be reversed by increasing pump output.

\section{Case report}

In 1972 a 24-year-old woman underwent mitral valve replacement with a Lillehei-Kaster prosthesis. Her mitral valve was a fixed, stenotic diaphragm unsuitable for valvotomy. When she was routinely reassessed 18 months later, as part of a study of Lillehei-Kaster valves, the prosthetię valve was thought at angiography to be functioning normally, although a mean diastolic gradient of $12 \mathrm{mmHg}$ across the valve orifice was noted which was considered to be high. The patient waso however, asymptomatic.

Eighteen months later she returned to hospitæ 35 weeks pregnant, with a history of having bee $\vec{B}$ unwell for the preceding six days with urgen? dyspnoea, palpitations, cough, and ankle oedema?

On examination there was tachycardia, hypo? tension $(85 / 60 \mathrm{mmHg})$, and a raised jugula venous pressure. There was a $3 / 6$ pansystolie murmur, which radiated to the axilla, and tripfe rhythm. The electrocardiogram (ECG) showe sinus rhythm with an axis of $+60^{\circ}$, a P-R intervai of $0.24 \mathrm{sec}$, and depressed ST segments in leads V1 to V6. A diagnosis of an obstructed disc valve was made.

Shortly after admission vaginal bleeding wass noticed and an obstetrician was consulted. Pre parations were made for urgent cardiopulmonary bypass for replacement of the clotted valve. The diagnosis of placenta praevia was made. At this juncture the fetal heart was still audible and the presentation was cephalic.

As a preliminary to the induction of anaesthesia with a view to Caesarean section, arterial and central venous pressure lines were introduced under local anaesthesia, and arterial blood preș sure, central venous pressure, pulse rate, and EC.G were continuously monitored. The fet@ heart was again thought to be audible.

Anaesthesia was then induced with the patie slightly laterally tilted, with a small dose of ketæ. 
mine and suxamethonium, followed by rapid intubation of the trachea. When the lower uterine segment was incised, maternal blood pressure, always low, fell further and an isoprenaline infusion was started. A fresh stillborn child was delivered and, because the mother's condition had now seriously deteriorated, an attempt was made rapidly to establish femorofemoral bypass. Cardiac arrest occurred before the groin vessels had been cannulated and attempts at resuscitation failed.

\section{Comment}

In an active cardiac surgical unit, painfully aware of the rapidity with which patients deteriorate when prosthetic valves become clotted and obstructed, and experienced in the rapid establishment of cardiopulmonary bypass for further valve replacement, the recognition and management of this common complication of valve surgery did not present a problem. However, when we were faced with this well recognised problem, further complicated by vaginal bleeding related to type 1 placenta praevia, there arose the need to establish priorities.

The pregnancy was valuable to the mother and the fetus was viable. Heparinisation was thought likely to precipitate massive bleeding and this, in an acutely ill patient, was judged likely to be fatal. Caesarean section, necessary for maternal and fetal survival, was therefore given priority.

In retrospect, a more logical course would have been to autotransfuse the patient with the blood which may have accumulated in the peritoneal cavity at Caesarean section. Autotransfusion in obstetrics is well documented. Highmore (1874) described its use in postpartum haemorrhage, while Miller (1973) discussed autotransfusion in the management of ectopic pregnancy.

Stehling et al. (1975) described the indications for and contraindications to the use of this technique. The indications listed were blunt or penetrating trauma to the chest or abdomen, ruptured ectopic pregnancy, elective surgery associated with large blood loss, and rare blood types or antibody peculiarities complicating the cross-matching of blood. The contraindications to the use of autotransfusion were gastrointestinal tract contamination, concomitant distant fractures or cerebral trauma in respect of the use of intravenous heparin, and the presence of cancer cells in the operative field.

As a precaution against microemboli-induced pulmonary changes, a $20-40$ micron filter should be included when using autotransfusion. Prolonged use of suction should also be avoided, since further destruction of the blood occurs. In units not accustomed to the use of cardiopulmonary bypass, the stringent precautions necessary to avoid air embolism should be carefully observed.

Faced with this problem again, we would establish femorofemoral bypass with its attendant necessity for heparinisation. The fetus would be carefully monitored by recording fetal heart rate, ECG, and the acid-base status of the mother. In our case, the excessive bleeding would have been managed by means of autotransfusion until Caesarean section was performed, followed by replacement of the obstructed mitral prosthesis.

\section{References}

Barnard, P. M.. Heydenrych, J. J.. and Lembaard, B. G. (1969). Mitral valve prosthesis and pregnancy without anticoagulation therapy. South African Medical Journal, 43, 1397-1398.

Bennett, G. G. and Oakley, C. M. (1968). Pregnancy in a patient with mitral-valve prosthesis. Lancet. 1, 616-619.

Brock. R. C. (1952). Valvotomy in pregnancy. Proceedings of the Royal Society of Medicine, 45, 538-540.

Buxbaum, A., Aygen. M. M.. Shahin, W.. Levy, M. J., and Ekerling, B. (1971). Pregnancy in patients with prosthetic heart valves. Chest, 59, 639-642.

Highmore, W. (1874). Practical remarks on an overlooked source of blood-supply to transfusion in post-partum haemorrhage. Lancet. 1, 89.

Koh, K. S., Friesen, R. M. Livingstone, R. A., and Peddle, L. J. (1975). Fetal monitoring during maternal cardiac surgery with cardiopulmonary bypass. Canadian Medical Association Journal, 112, 1102-1104.

Laros, R. K., Jr., Hage, M. L., and Hayashi, R. H. (1970). Pregnancy and heart prostheses. Obstetrics and Gynecology, 35, 241-247.

Logan, A. and Turner, R. (1952). Mitral valvulotomy in pregnancy. Lancet, 1, 1286.

Miller, R. D. (1973). Complicaticns of massive blood transfusions. Anesthesiology. 39, 82-93.

Reid, J. M. and Barclay, R. S. (1971). Pregnancy and mitral valve prosthesis. British Medical Jonrnal, 1, 326-327.

Stehling, L. C., Zauder, H. L.. and Rogers, W. (1975). Intraoperative autotransfusion. Anesthesiology, 43, 337-345.

Zitnik, R. S.. Brandenburg. R. O., Sheldon, R., and Wallace. R. B. (1969). Pregnancy and cpen-heart surgery. Circulation, 39/40, Supplement, 257-262.

Requests for reprints to: Professor B. T. le Roux, Department of Thoracic Surgery, Wentworth Hospital, P.B. Jacobs, Durban, Natal 4026, South Africa. 